\title{
Blue Gene: A Massively Parallel System
}

\author{
Jose E. Moreira \\ Research Staff Member \\ IBM T. J. Watson Research Center \\ Yorktown Heights NY 10598-0218 \\ phone: +1-(914)-945-3987 \\ fax: +1-(914)-945-4425 \\ jmoreira@us.ibm.com
}

\begin{abstract}
Blue Gene is a massively parallel system being developed at the IBM T. J. Watson Research Center. With its 4 million-way parallelism and 1 Petaflop peak performance, Blue Gene is a unique environment for research in parallel processing. Full exploitation of the machine's capability requires 100-way shared memory parallelism inside a single-chip multiprocessor node and message-passing across 30,000 nodes. New programming models, languages, compilers, and libraries will need to be investigated and developed for Blue Gene, therefore offering the opportunity to break new ground in those areas. In this talk, I will describe some of the hardware and software features of Blue Genes. I will also describe some of the protein science and molecular dynamics computations that are important driving forces behind Blue Gene.
\end{abstract}

